\title{
Evaluation of Tree Species Classification Methods using Multi-Temporal Satellite Images
}

\author{
Arnav Saha ${ }^{l}$, Srikumar Sastry ${ }^{l}$, Viral A. Dave ${ }^{l,}{ }^{*}$, Ranendu Ghosh ${ }^{1, *}$ \\ ${ }^{1}$ Dhirubhai Ambani Institute of Information and Communication Technology, Gandhinagar, India \\ *viral_dave@daiict.ac.in,ranendu_ghosh@daiict.ac.in
}

\begin{abstract}
Tree species classification is an important step towards forest monitoring and biodiversity conservation. This research study evaluates several multispectral image classification techniques for tree species over Ahwa village in Dang district, South Gujarat, India. Multispectral images consisting of 4 bands-R, G, B and NIR collected over 4 months was used. Object-based segmentation using mean shift, cluster-based using K-Means and Gaussian Mixture Model (GMM) and pixel-based methods have been analyzed. Additionally, a new method of classification has been described using the Dynamic Time Warping (DTW) algorithm. It outperformed supervised classification techniques with accuracy over $95 \%$. The GMM+DTW model accurately reflected the actual species distribution found in the ground truth.
\end{abstract} DTW

Index Terms - Multispectral, Temporal matching,

\section{INTRODUCTION}

Remote sensing has been widely recognized for its contributions towards monitoring the change in land cover vegetation, ecological studies, mapping biodiversity etc. for quite some time now. It is important to study species diversity of an area as it is an indicator of its overall ecological health. However, species monitoring over a large area is a monotonous task even for experts. The use of multispectral imagery for the monitoring of land cover vegetation has received recognition from forest monitoring agencies lately. The aim of this paper is to evaluate multiple classification techniques. Section 2 describes the study area and data preprocessing. Section 3 explains the methodology and Section 4 describes the obtained results and conclusion.

\section{STUDY AREA AND DATASET}

Ahwa, the district capital of the Dangs was chosen as the study area (SA). It is a hilly region with thick forest situated in Southern Gujarat, India between $20.748^{\circ} \mathrm{N}, 73.677^{\circ} \mathrm{E}$ and $20.766^{\circ} \mathrm{N}, 73.697^{\circ} \mathrm{E}$. The forestland predominantly falls under the reserve forests and have been labelled as Tropical Moist Deciduous with Teak (tectona grandis), Sadad (terminalia tomentosa), Sisam, Khair etc. as its major tree species. High resolution $(5 \mathrm{~m})$ multispectral temporal images over the SA were selected over four dates, one from each month of December 2018, (January, February and March) 2019.

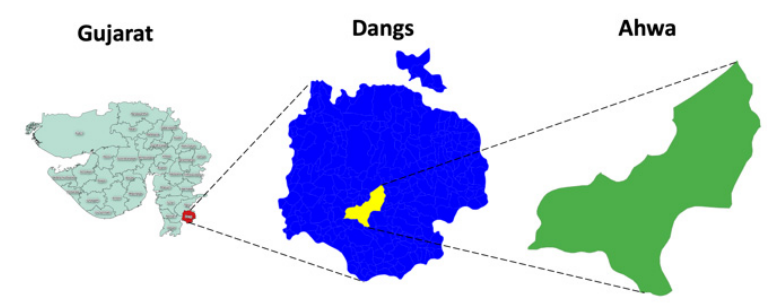

Fig. 1. The study area located in South Gujarat.

The available dataset was already geo-rectified and orthorectified. The non-forest components such as roads, water bodies, crops etc. needed to be masked out. The mask provided by Forest Survey of India (FSI) was used. It was applied on all the bands of all temporal images. For clustering and segmentation, all the temporal images were stacked together to form a 16-band composite stacked vector [1]. These 16 bands acted as a feature vector for each pixel. This stacked vector included the temporal variation information of each pixel which is an important criterion for clustering. All the data pre-processing was done using QGIS v2.7.
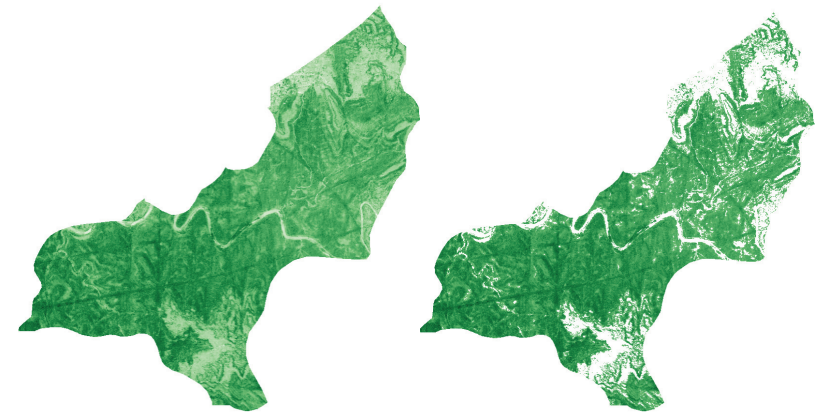

Fig. 2. FSI mask applied on Ahwa to retain forest cover. The white area represents the non-forest area. 


\section{METHODOLOGY}

The methodology involves the following steps: (1) data processing to create clusters using kmenas and gmm and objects using mean shift segmentation to compile NDVI profiles of the clusters; (2) data classification using DTW by matching NDVI profiles with pure profiles and supervised algorithms like RF and DT (3) Evaluation of all the methods using GT samples to determine their accuracy.

\subsection{Clustering the stacked vector (cluster-based method)}

Clustering algorithms [2] group data points into subsets having similar properties. These subsets can then be used for supervised classification as opposed to individual data point classification. The obtained stacked vector was clustered using two of the most popular clustering algorithms, namely K-Means [3] and Gaussian Mixture Model (GMM) [4]. As the stacked vector was large in terms of the number of pixels, other clustering algorithms like hierarchical clustering and spectral clustering [5] were computationally infeasible. The sole purpose of using various clustering algorithms was to evaluate their performance on multispectral images and proceeding with the best one.
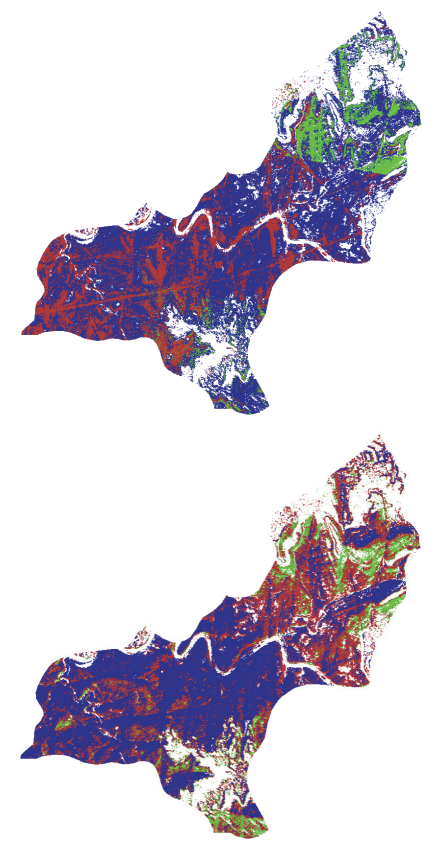

Fig. 3. Clustered images with GMM (top) and K-Means (bottom) using three clusters.

Both K-Means and GMM have some parameters which need to be tuned for optimal performance. But the most important parameter common to them is the number of components. To decide upon the number of clusters and analyze its corresponding performance, silhouette score [6] is used as a metric. Silhouette score gives a measure of average similarity between a data point and its cluster. The K-Means is hard in nature while GMM is soft in nature. The GMM gives a better performance over raw data and when the data is not spherical [7]. According to Table 1, GMM with 3 clusters was chosen.

\section{Table 1}

Performance measure for the two clustering algorithms on varying number of clusters.

\begin{tabular}{cccc}
\hline & \multicolumn{3}{c}{ Silhouette Score } \\
\cline { 2 - 4 } Method & 3 & 4 & 5 \\
\cline { 2 - 4 } Number of clusters \\
\hline K-Means & 0.78 & 0.75 & 0.72 \\
GMM & 0.85 & 0.76 & 0.73 \\
\hline
\end{tabular}

\subsection{Segmentation of multi-temporal images (object- based method)}

Clustering works well for the analysis of unlabeled datapoints, but fails to retain the spatial information contained in the image. Moreover, it is hard to detect edges in clustered images. Pinaki [8] concluded that Multi Resolution Segmentation and Watershed perform poorly on forest areas due to over estimation while Meanshift performs well. So, the Meanshift segmentation was applied on temporal images using the 16 bands as input variable (fig. 4). It primarily serves to find homogeneity in unclassified data by dividing it into segments.

\subsection{Temporal Profiles}

The temporal profiles of individual pixels and the clusters were calculated using NDVI (Normalised Difference Vegetation Index). NDVI is calculated using:

$$
\mathrm{NDVI}=\frac{\mathrm{NIR}-\mathrm{R}}{\mathrm{NIR}+\mathrm{R}}
$$

NDVI [9] is one of the most popular indexes used for studying vegetation type and its health. NDVI is able to quantify phenological differences among vegetation types [10]. It reduces noise due to varying sun angles, clouds, atmospheric conditions and topography [11]. According to Tucker et al. [12], green-leaf biomass is an important criterion for species separation. It varies with season and is effectively quantified by NDVI. The chosen classes for classification (based on ground truth) Teak (tectona grandis), Sadad (terminalia tomentosa) and Mixed forest (includes both teak, sadad and other species) exhibit different phenological and physical characteristics. Teak is a deciduous species which can grow up to $40 \mathrm{~m}$ in height. Its flowering season starts in June and end in August. Fruiting occurs from September to December. Sadad can grow up to $30 \mathrm{~m}$ in height. Flowering and fruiting period of Sadad is from April-May. This tree has a unique property of holding water 


\section{DTW}

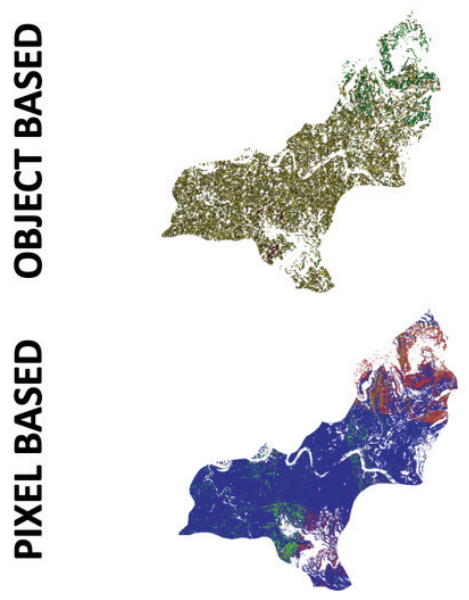

RANDOM FOREST
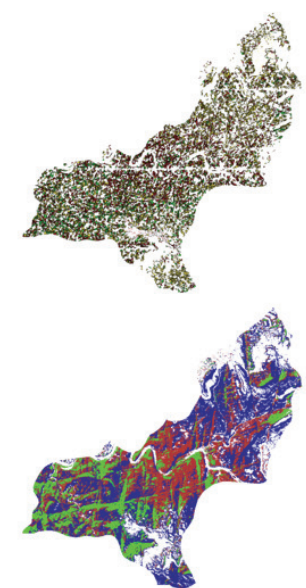

DECISION TREE
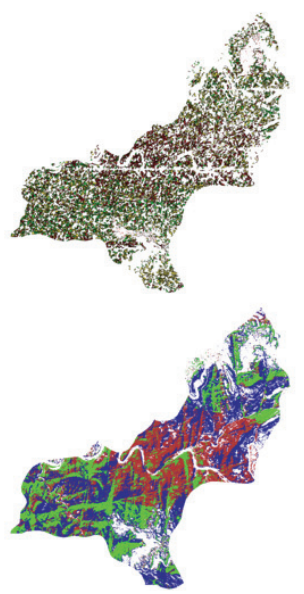

Fig. 4. Classified images using object-based and pixel-based methods. The white area represents the non-forest mask.

in dry seasons. Sadad can be visually separated from Teak due to its wide canopy, elliptical leaves and greyish barks. As a result of these characteristics, each one exhibits a different temporal profile. The pure profiles of the trees were obtained from ground truth conducted in the forests.

\section{NDVI profile of clusters}

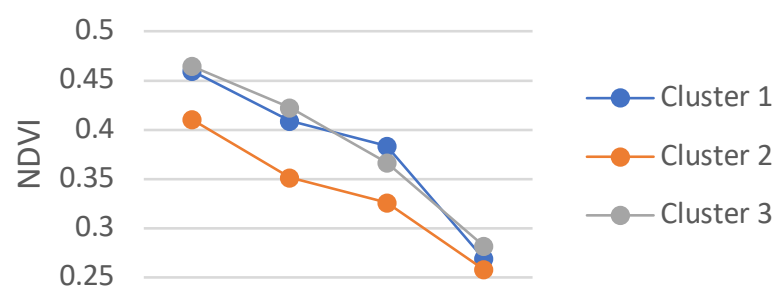

Dec'18 Jan'19 Feb'19 Mar'19

Pure profiles of the classes

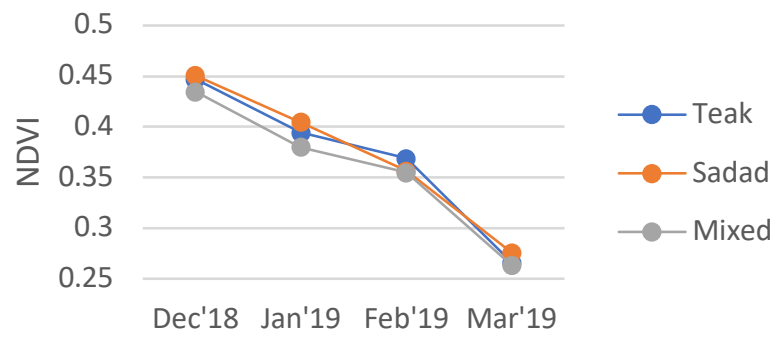

Fig. 5. Temporal NDVI profiles generated from clusters (top) and pure profiles of dominant species (bottom).

The temporal profile of the clusters/pixels will not change much year by year as most of the forest areas are restricted/protected i.e., local intervention is strictly prohibited. They are subject to change only in adverse climatic or atmospheric conditions. The regularity in temporal signatures year by year is important as the classification algorithm now does not have to deal with such pattern changes. This proves to be an advantage over some domains such as crop mapping where temporal signatures might change yearly depending upon agricultural practices and rainfall [13] and land use land cover (LULC) mapping where irregularities in phenological patterns cannot be dealt with by classification methods [14].

\subsection{Temporal Matching using DTW}

Dynamic Time Warping (DTW) is a time series analysis algorithm which computes the dissimilarity between two time series datasets [15]. For the cluster-based approach, the pure patterns from the ground truth were matched with the temporal patterns of the clusters (fig. 5). Each cluster was assigned a class based on the DTW output (fig. 3). For the object-based approach, profile of individual objects was constructed by computing the mean of the bands of all pixels within the object. This profile was then matched with the pure profiles. Similar approach was taken for the pixel-based (PW) method.

\subsection{Random Forest and Decision Tree}

Random forest and decision tree were trained on the ground samples which were used for generating the pure profiles. All 16 bands were used as the input variable. Both the classifiers were used for object-based and pixel-based classification. (fig. 4).

\section{RESULTS AND DISCUSSION}

The performance of each of the models was evaluated by calculating the Overall accuracy (OA) and the Kappa score. The models were validated on 6 ground truth samples taken inside the study area. These sites were randomly sampled to avoid bias. Table 2 clearly shows that DTW produced higher 
accuracy and Kappa scores with GMM+DTW being the best estimator.

\section{Table 2}

Overall accuracy and Kappa score of the models applied to the ground truth samples.

\begin{tabular}{ccc}
\hline Classification models & OA (\%) & Kappa \\
\hline GMM+DTW & 94.54 & 0.88 \\
MS+RF & 87.67 & 0.72 \\
MS+DT & 87.50 & 0.74 \\
MS+DTW & 91.11 & 0.81 \\
PW-DTW & 86.32 & 0.72 \\
PW-RF & 86.47 & 0.70 \\
PW-DT & 85.91 & 0.69 \\
\hline
\end{tabular}

Overall, both Mean shift and GMM produced acceptable results. However, only GMM+DTW accurately mapped the tree species distribution across Ahwa.

Traditionally, segmentation was applied on single date image [16] but in our case, clustering and segmentation on multi date image proved to be efficient. It takes into account the temporal variation and possibly removes salt and pepper noise associated with a group of pixels. If temporal images of several dates are made available at high resolution, the potential of forest monitoring and conservation will grow endlessly.

\section{REFERENCES}

[1] P. Mather, Computer processing of remotely-sensed images: an introduction, New York: John Wiley \& Sons, 1988.

[2] L. Rokach, "A survey of Clustering Algorithms," in Data Mining and Knowledge Discovery Handbook, Springer, 2010, pp. 269-298.

[3] V. Faber, "a Clustering and the Continuous k-means Algorithm," o Los Alamos Science, vol. 22, pp. 138$144,1994$.

[4] S. Dasgupta, "Learning mixtures of Gaussians," in IEEE Symp. on Foundations of Computer Science, New York, 1999.

[5] C. J. Alperta, A. B. Kahn and S.-Z. Yao, "Spectral partitioning with multiple eigenvectors," Discrete Applied Mathematics, vol. 90, 1999.
[6] P. J.Rousseeuw, "Silhouettes: A graphical aid to the interpretation and validation of cluster analysis," Journal of Computational and Applied Mathematics, vol. 20, pp. 53-65, 1987.

[7] Z. Wang, C. d. Cunha, M. Ritou and B. Furet, "Comparison of K-means and GMM methods for contextual clustering in HSM," Procedia Manufacturing, no. 28, pp. 154-159, 2019.

[8] P. R. Sarkar, "Comparison of Segmentation Algorithms and Estimation of Optimal Segmentation Parameters for Very High Resolution Satellite Imagery," Indian Institute of Space Science and Technology, Secunderabad, 2016.

[9] C. Tucker, "Red and photographic infrared linear combinations for monitoring vegetation," Remote Sens. Environ, pp. 127-150, 1979.

[10] R. Defries and J. Townshend, "NDVI-Derived Land Cover Classification at a Global Scale," International Journal of Remote Sensing - INT J REMOTE SENS, 1994.

[11] A. Huete, K. Didan, T. Miura, E. Rodriguez, X. Gao and L. Ferrerira, "Overview of the radiometric and biophysical performance of the MODIS vegetation indices," Remote Sens. Environ., pp. 195-213, 2002.

[12] C. Tucker, "Analysis of the phenology of global vegetation using meteorological satellite data," International Journal of Remote Sensing, 1985.

[13] F. Petitjean, J. Inglada and P. Gançarski, "Satellite image time series analysis under time warping," IEEE Trans. Geosci. Remote Sens., pp. 3081-3095, 2012.

[14] V. Maus, G. Camara, R. Cartaxo, A. Sanchez, F. Ramos and G. Queiroz, "A time-weighted dynamic time warping method for land-use and land-cover mapping," IEEE J. Select. Top. Appl. Earth Observ. Remote Sens, pp. 1-11, 2016.

[15] H. Sakoe and S. Chiba, "Dynamic programming algorithm optimization for spoken word recognition," IEEE Trans. Acoust. Speech Signal Process, pp. 43-49, 1978.

[16] B. Desclée, P. Bogaert and P. Defourny, "Forest change detection by statistical object-based method," Remote Sens. Environ, pp. 1-11, 2006. 